\title{
Article \\ Xylitol Inhibits Growth and Blocks Virulence in Serratia marcescens
}

\author{
Ahdab N. Khayyat ${ }^{1}$, Wael A. H. Hegazy ${ }^{2} *^{\mathbb{D}}$, Moataz A. Shaldam ${ }^{3} \mathbb{D}$, Rasha Mosbah ${ }^{4,5}$ MD Ahmad J. Almalki ${ }^{1}{ }^{\mathbb{D}}$, \\ Tarek S. Ibrahim ${ }^{1,6} \mathbb{D}^{\mathbb{D}}$, Maan T. Khayat ${ }^{1}$, El-Sayed Khafagy ${ }^{7,8} \mathbb{D}^{\mathbb{D}}$, Wafaa E. Soliman ${ }^{9,10} \mathbb{D}$ and Hisham A. Abbas ${ }^{2} \mathbb{D}$
}

check for updates

Citation: Khayyat, A.N.; Hegazy, W.A.H.; Shaldam, M.A.; Mosbah, R.; Almalki, A.J.; Ibrahim, T.S.; Khayat, M.T.; Khafagy, E.-S.; Soliman, W.E.; Abbas, H.A. Xylitol Inhibits Growth and Blocks Virulence in Serratia marcescens. Microorganisms 2021, 9 , 1083. https://doi.org/10.3390/ microorganisms 9051083

Academic Editor: Giuseppantonio Maisetta

Received: 18 April 2021

Accepted: 12 May 2021

Published: 18 May 2021

Publisher's Note: MDPI stays neutral with regard to jurisdictional claims in published maps and institutional affiliations.

Copyright: (c) 2021 by the authors. Licensee MDPI, Basel, Switzerland. This article is an open access article distributed under the terms and conditions of the Creative Commons Attribution (CC BY) license (https:/ / creativecommons.org/licenses/by/ $4.0 /)$.
1 Department of Pharmaceutical Chemistry, Faculty of Pharmacy, King Abdulaziz University, Jeddah 21589, Saudi Arabia; Ankhayyat@kau.edu.sa (A.N.K.); Ajalmalki@kau.edu.sa (A.J.A.); tmabrahem@kau.edu.sa (T.S.I.); mkhayat@kau.edu.sa (M.T.K.)

2 Department of Microbiology and Immunology, Faculty of Pharmacy, Zagazig University, Zagazig 44519, Egypt; hishamabbas2008@gmail.com

3 Department of Pharmaceutical Chemistry, Faculty of Pharmacy, Kafrelsheikh University, Kafr El-Sheikh 33516, Egypt; dr_moutaz_986@yahoo.com

4 Infection control Unit, Zagazig University Hospitals, Zagazig University, Zagazig 44519, Egypt; Rashamosbah6@gmail.com

5 Faculty of Oral and Dental medicine, Ahram Canadian University, Giza Governorate 12573, Egypt

6 Department of Pharmaceutical Organic Chemistry, Faculty of Pharmacy, Zagazig University, Zagazig 44519, Egypt

7 Department of Pharmaceutics, College of Pharmacy, Prince Sattam Bin Abdulaziz University, Al-kharj 11942, Saudi Arabia; e.khafagy@psau.edu.sa

8 Department of Pharmaceutics and Industrial Pharmacy, Faculty of Pharmacy, Suez Canal University, Ismailia 41552, Egypt

9 Department of Biomedical science, Faculty of Clinical Pharmacy, King Faisal University, Alhofuf, Al-Ahsa 36362, Saudi Arabia; wafaaezz2006@yahoo.com

10 Department of Microbiology and Biotechnology, Faculty of Pharmacy, Delta University for Science and Technology, Mansoura 11152, Egypt

* Correspondence: waelmhegazy@daad-alumni.de; Tel.: +20-1101188800

\begin{abstract}
Serratia marcescens is an opportunistic nosocomial pathogen and causes wound and burn infections. It shows high resistance to antibiotics and its pathogenicity is mediated by an arsenal of virulence factors. Another therapeutic option to such infections is targeting quorum sensing (QS), which controls the expression of different S. marcescens virulence factors. Prevention of QS can deprive $S$. marcescens from its bacterial virulence without applying stress on the bacterial growth and facilitates the eradication of the bacteria by immunity. The objective of the current study is to explore the antimicrobial and antivirulence activities of xylitol against $S$. marcescens. Xylitol could inhibit the growth of $S$. marcescens. Sub-inhibitory concentrations of xylitol could inhibit biofilm formation, reduce prodigiosin production, and completely block protease activity. Moreover, xylitol decreased swimming motility, swarming motility and increased the sensitivity to hydrogen peroxide. The expression of $r s m A$, pigP, flhC, flhD fimA, fimC, shlA $b s m B$, and $r s s B$ genes that regulate virulence factor production was significantly downregulated by xylitol. In silico study showed that xylitol could bind with the SmaR receptor by hydrophobic interaction and hydrogen bonding, and interfere with the binding of the natural ligand with SmaR receptor. An in vivo mice survival test confirmed the ability of xylitol to protect mice against the virulence of $S$. marcescens. In conclusion, xylitol is a growth and virulence inhibitor in $S$. marcescens and can be employed for the treatment of $S$. marcescens wound and burn infections.
\end{abstract}

Keywords: Serratia marcescens; quorum sensing; virulence; xylitol

\section{Introduction}

Serratia marcescens is an environmental rod-shaped gram-negative bacterium that belongs to the Enterobacteriaceae family. S. marcescens is an opportunistic microbe that 
can cause several nosocomial infections [1]. Moreover, S. marcescens can cause wound and soft tissue infections [2]. Considerably, S. marcescens is responsible for $11 \%$ of open burnrelated surgical wound infections [3], and is one of the gram-negative bacteria that causes invasive burn wound infections [4]. S. marcescens' pathogenicity is attributed to its arsenal of virulence factors including protease, nuclease, lipase and hemolysin in addition to its ability to swarm and swim and to form biofilm [5]. S. marcescens produces a red pigment, prodigiosin, which possesses different activities against fungi, bacteria and protozoa [6,7]. Moreover, prodigiosin has immunosuppressive effects [8].

The treatment of $S$. marcescens infections is hindered by its multidrug-resistant nature. It has showed resistance to fluoroquinolones, aminoglycosides and $\beta$-lactams [9]. Furthermore, $S$. marcescens can adhere to medical instruments forming biofilms. Biofilm formation adds to the problem of antibiotic resistance by forming a protective shield for microorganisms against antibiotics [1]. Using antibiotics poses a selective growth stress on bacteria that leads to the emergence of bacterial resistance. This is why there is an increasing demand to develop alternative strategies. One of them is targeting the intercellular communication system, quorum sensing (QS), which helps bacteria to survive in stressed conditions [5]. QS depends on secretion of signaling molecules or auto-inducers that regulate the expression of virulence genes in bacterial densities when they reach a certain threshold of concentration $[7,10,11]$. In $S$. marcescens, QS regulates the expression of several virulence factors including nuclease, hemolysin, lipase, protease, prodigiosin, biofilm formation and motility [12]. QS inhibitors pave the way to a new trend in treating $S$. marcescens infections, either alone or in combination with antibiotics $[5,13]$. This approach does not affect bacterial growth, and development of resistance is much less likely to occur. Moreover, inhibition of QS can enhance the immune response against bacteria [14,15].

The use of sugars such as sucrose and hypertonic glucose has been reported as a bacterial growth inhibitor. The application of sugars to the wound provides a local osmotic effect that enhances the formation of granulation tissue, reduces edema in wounds, and leads to improvement of microcirculation of the wound. As a result, the healing time of the patient is reduced and the needed medical care is lessened [16-18]. Xylitol is a sugar alcohol that was previously found to have antimicrobial activity against Streptococcus pneumoniae and Streptococcus mutants [19]. In addition, xylitol could inhibit the adhesion of many bacteria [20]. The application of xylitol in wound care was reported in an in vitro Lubbock Chronic Wound Biofilm model, where the growth of Staphylococcus aureus, Pseudomonas aeruginosa, and Enterococcus faecalis was reduced by the use of $2 \%, 10 \%$, and $20 \%$ xylitol in water and biofilm formation was completely blocked by $20 \%$ xylitol [21]. Moreover, combining 2\% lactoferrin with 5\% xylitol decreased the biofilm formation in methicillin-resistant $S$. aureus and $P$. aeruginosa as compared to base wound dressings alone [22]. Furthermore, the negative dissolution energy of xylitol exerts a cooling effect on the inflamed tissues [22,23].

In our previous study, xylitol could inhibit biofilms of $P$. aeruginosa clinical isolates [24]. Interestingly, xylitol was found to possess antivirulence activity against the model bacterium Chromobacterium violaceum CV026 by affecting QS [23]. However, the activity of xylitol on the growth, QS and virulence in S. marcescens has not been previously studied, to our knowledge. In this study, the antimicrobial and antivirulence effects of xylitol on $S$. marcescens were investigated.

\section{Materials and Methods}

\subsection{Chemicals and Microbiological Media}

All chemicals were of pharmaceutical grade; these include microbiological media, Tryptone soya broth and agar, Luria-Bertani (LB) broth and agar, and Mueller Hinton broth. Xylitol was ordered from Sigma-Aldrich (St. Louis, MO, USA). 


\subsection{Bacterial Strains}

The $S$. marcescens clinical isolate used in the current study was obtained from a patient that was admitted to the Intensive Care Unit at Zagazig University Hospital from a surgical wound infection. This isolate was fully identified by the MALDI-TOF apparatus $[7,10,25]$.

\subsection{Determination the Minimum Inhibitory Concentration (MIC) of Xylitol}

To determine the MIC of xylitol, the broth microdilution method was employed (CLSI, 2015), and MIC was determined as the least concentration of xylitol with which no visible bacterial growth was observed.

\subsection{Evaluation of Biofilm Production}

For assessment of biofilm production, S. marcescens overnight cultures were cultivated, diluted with tryptone soya broth (TSB) and the optical density was adjusted to a cell density of $1 \times 106 \mathrm{CFU} / \mathrm{mL}[10,26]$. Aliquots of $200 \mu \mathrm{L}$ of the prepared bacterial suspension were transferred in sterile 96 -well polystyrene microplates and incubated overnight at $37^{\circ} \mathrm{C}$. The non-adherent planktonic cells were aspirated and the wells were washed. The adherent cells were fixed with methanol (99\%) for $25 \mathrm{~min}$, and stained with crystal violet (1\%) for $25 \mathrm{~min}$. The unattached dye was washed off, and the plates were air-dried. The bounded crystal violet dye was extracted by 33\% glacial acetic and absorbance was measured with $95 \%$ ethanol. The test was repeated in triplicate, the mean optical densities were calculated at a wavelength of $590 \mathrm{~nm}$, and the cut-off optical density (ODc) was calculated. The cut-off $\mathrm{OD}(\mathrm{ODc})$ is defined as three times standard deviations above the mean OD of the negative control. The tested isolate was categorized into one of four groups; non-biofilm forming $(\mathrm{OD} \leq \mathrm{ODc})$, weak-biofilm forming (OD $>$ ODc, but $\leq 2 \times$ ODc), moderate-biofilm forming (OD $>2 \times$ ODc, but $\leq 4 \times \mathrm{ODc}$ ), or strong-biofilm forming (OD $>4 \times \mathrm{ODc}$ ).

\subsection{Biofilm Inhibition Assay}

The xylitol inhibitory activity on the biofilm formation was assessed as indicated formerly $[15,25,27,28]$. The xylitol inhibitory effects were evaluated at different concentrations $(5 \%$ and $10 \%)$ and the biofilm inhibition was calculated and presented as a percentage of the control free from xylitol.

Moreover, the biofilm inhibition by xylitol was visualized under light microscope $[7,29]$. The formed biofilms on cover slips in presence or absence of xylitol $(5 \%$ or $10 \%)$ were stained with crystal violet and imaged under light microscope.

\subsection{Prodigiosin Inhibition Assay}

The ability of xylitol to interfere with the prodigiosin production of $S$. marcescens was evaluated as previously shown $[7,10,25]$. The prodigiosin dye was extracted and acidified from bacterial cultures that were treated with or without $5 \%$ or $10 \%$ xylitol, and the absorbance was estimated at $534 \mathrm{~nm}$ via Biotek Spectrofluorometer (Biotek, Winooski, VT, USA). The prodigiosin inhibition was expressed as a percentage of the untreated xylitol-free control.

\subsection{Protease Assay}

The proteolytic activity of the tested $S$. marcescens was assessed in the absence or presence of $5 \%$ or $10 \%$ xylitol by the skim milk agar method $[7,15]$. The supernatants containing extracellular protease collected from treated bacterial cultures provided with or without xylitol were collected and added to wells in 5\% skim milk LB agar plates. After incubation, the formed clear zones due to protease activity were measured. The percentage of protease inhibition was shown as a percentage of the xylitol-free control.

\subsection{Assay of Bacterial Motilities}

S. marcescens motility assays were performed in the presence or absence of $5 \%$ or $10 \%$ xylitol $[7,15,25,30]$. The LB $0.3 \%$ or $0.5 \%$ agar plates with or without $5 \%$ or $10 \%$ xylitol were prepared, and centrally cultured with $S$. marcescens for swimming or swarming motilities 
assays, respectively. The swimming or swarming zones in xylitol containing plates were measured in $\mathrm{mm}$ and presented in comparison to control xylitol-free plates.

\subsection{Oxidative Stress Assay}

In order to investigate the ability of xylitol to restrict the $S$. marcescens resistance to oxidative stress, the oxidative stress was assessed in presence of xylitol [15,31]. Tryptone soya agar (TSA) plates with xylitol (5\% and 10\%) and control plates without xylitol were prepared. S. marcescens isolate was grown overnight in TSB and $100 \mu \mathrm{L}$ from the culture were spread on the surface of the plates. Ten microliters of $3 \% \mathrm{H}_{2} \mathrm{O}_{2}$ were added to sterile filter-paper disks on the surface of plates. After overnight incubation, the inhibition zones due to $\mathrm{H}_{2} \mathrm{O}_{2}$ were measured.

\subsection{Quantification of Virulence Genes' Expressions}

In order to explore the influence of xylitol on expression of virulence genes, the RNA was extracted from $S$. marcescens cultures treated or untreated with xylitol (5\%) using RNeasy Mini kit (Qiagen, Hilden, Germany). The extracted RNA concentrations were quantified by NanoDropTM 2000 (Thermo Fisher Scientific, Waltham, MA, USA). A Reverse Transcriptase kit (Thermo Fisher Scientific, Waltham, MA, USA) was employed to synthesize cDNA from extracted RNA samples. The cDNA amplification was performed with the SYBR Green Quantitative RT-qPCR Kit (Thermo Fisher Scientific, Waltham, MA, USA). The expression levels of virulence genes fim $A, \operatorname{fim} C, \operatorname{rss} B, b s m B, \operatorname{rsm} A$, shlA, pigB, flhC and $f l h D$ were standardized in relation to the critical threshold (CT) mean values of rplU as a housekeeping gene by the $2^{-\Delta \Delta C t}$ method [32-34]. The designed genes' primers and PCR cycles used in this study have been designated formerly $[7,25]$.

\subsection{Mice Survival Test}

The in vivo survival model was used to assess xylitol's protective activity against S. marcescens virulence [7,15,33,35]. Approximately, $1 \times 10^{8} \mathrm{CFU} / \mathrm{mL}$ S. marcescens treated with or without sub-MIC of xylitol $(100 \mathrm{mg} / \mathrm{mL})$ were suspended in phosphate-buffered saline. Twenty healthy female albino mice (Mus musculus) with the same age, 3-weeks old, and same weight were distributed in 4 groups. In the first group, mice were intraperitoneally injected with $100 \mu \mathrm{L}$ of xylitol-treated S. marcescens. In the second positive control group, mice were injected with untreated $S$. marcescens. In the third and fourth negative control groups, mice were either injected with $100 \mu \mathrm{L}$ of sterile PBS or kept uninoculated. All mice were kept in safe and suitable cages with normal feeding and aeration in the Faculty of Pharmacy animal house, Zagazig University, Egypt. The mice survival in all groups was noted every day for 5 consecutive days.

\subsection{In-Silico Analysis of Binding to SmaR Receptor}

The ability of xylitol to bind with the protein receptor SmaR was investigated by docking analysis. The SmaR protein 3D structure was created by SWISS-MODEL Server [36] using the crystal structure of the bacterial quorum-sensing transcription factor as a template (PDB code 1L3L) [37]. The study was carried out on xylitol and the co-crystalized natural ligand, C4HSL, in the receptor's active site using AutoDock Vina [38]. Ligand structures were sketched into Marvin Sketch V18.23.0 (Marvin Sketch, 2019), and the most energetically favored conformer was presented in format $\left({ }^{*} \cdot \mathrm{pdb}\right)$. All the rotatable bonds in ligands that were set to be flexible and the package of AutoDockTools was employed to detect Gasteiger atomic partial charges [39]. For preparation of the receptor, hydrogen atoms were included, Gasteiger atomic partial charges were determined, and the receptor and ligands were changed to the PDBQT format. In the configuration of AutoDock Vina, the parameter num modes were adjusted to 10 and exhaustiveness to 10 . The grid box center $(x=20.67, y=20.59$ and $z=20.06)$ with size $(x=13, y=13, z=13)$ was used to recognize the docking active site. Pymol (PyMOL Molecular Visualization System 2019) was used for 3D visualization and the 2D graphic presentation was created using LigPlot+ V1.4.5 [40]. 


\subsection{Statistical Analysis}

All assays were conducted in triplicate and the one-way ANOVA test (Graph Pad Prism 8) was employed for evaluation of the significance of xylitol's inhibitory activities on S. marcescens virulence factors. Statistical significance was considered when $p$ values $<0.05$.

\section{Results}

\subsection{Determination of MIC}

Xylitol could inhibit the growth of $S$. marcescens isolate at $400 \mathrm{mg} / \mathrm{mL}(40 \%$ concentration). Sub-inhibitory concentrations of xylitol 10\% (100 mg/mL) and $5 \%(50 \mathrm{mg} / \mathrm{mL})$ that are equivalent to $1 / 4$ and $1 / 8 \mathrm{MICs}$, respectively, were used for testing the inhibitory activities of xylitol against virulence of $S$. marcescens.

\subsection{Xylitol Inhibits Biofilm Formation}

For biofilm production assessment, the ODc and OD of tested S. marcescens isolate were measured, and it was considered strong-biofilm forming as its OD $>4 \times$ ODc. Biofilm formation was assayed in the absence and presence of xylitol, and xylitol was found to inhibit biofilm formation. Biofilm inhibition ranged between $74.22 \%$ and $81.03 \%$ at $5 \%$ and 10\% of xylitol, respectively (Figure 1B). Microscopic visualization of biofilm under the effect of xylitol was also performed by light microscopy. The microscopic examination revealed significant reduction in both the thickness of and surface area covered by the biofilms in xylitol's presence (Figure 1A).

A)

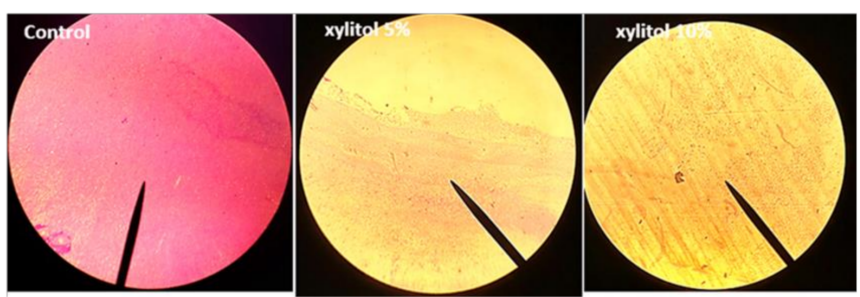

B)

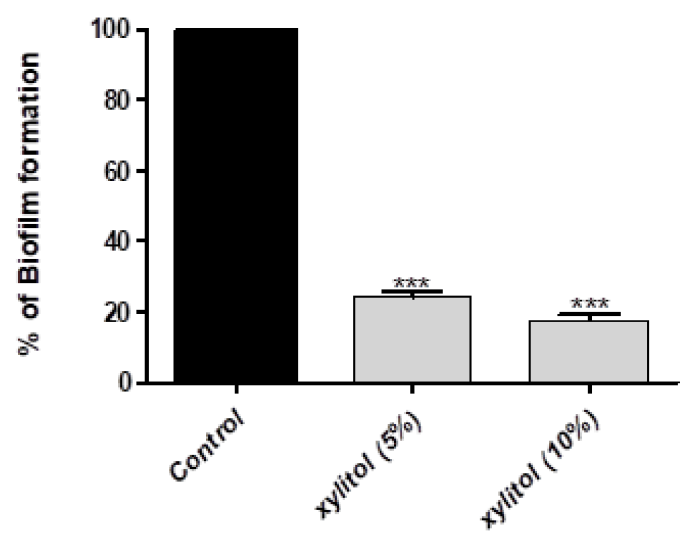

Figure 1. Biofilm inhibition of S. marcescens by xylitol. (A) Microscopic examination of biofilm inhibition by xylitol. Biofilm was formed on glass cover slips by xylitol-treated and untreated bacteria, and light microscopy was used for visualization. Both thickness and biofilm biomass were reduced by xylitol. (B) Biofilm was allowed to form on microtiter plate wells in the presence or absence of xylitol (5\% and $10 \%)$ and adherent cells were stained with crystal violet, glacial acetic acid was added to dissolve the dye, and the absorbance was measured at $590 \mathrm{~nm}$. The test was performed in triplicate. Biofilm formation was significantly reduced by xylitol. The data shown are the means \pm standard errors. One-way ANOVA test followed by Dunnett's Multiple Comparison test was used for statistical analysis. ${ }^{* * *}: p \leq 0.001$. 


\subsection{Xylitol Decreases Prodigiosin Production}

The red intracellular prodigiosin dye is produced by S. marcescens under the regulation of QS. Xylitol exhibited a marked inhibitory activity against prodigiosin production that reached $63.42 \%$ and $78.47 \%$ at concentrations $5 \%$ and $10 \%$ of xylitol, respectively (Figure 2).

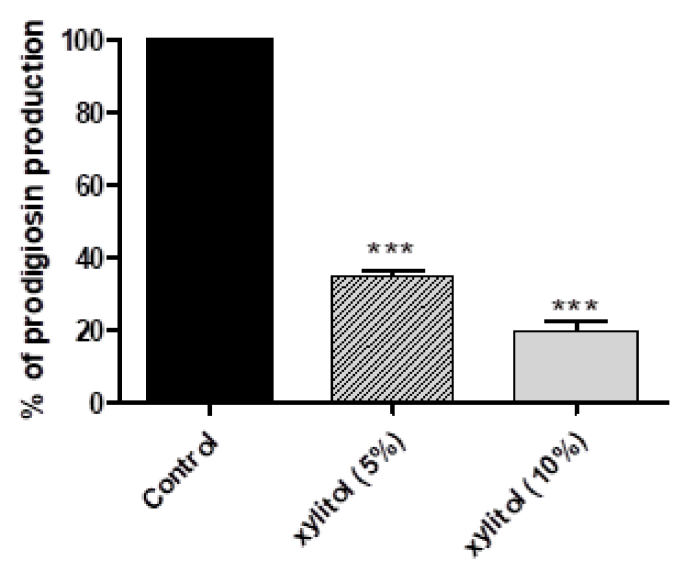

Figure 2. Inhibition of prodigiosin production by Serratia marcescens by xylitol. Prodigiosin pigment was extracted from xylitol-treated and untreated cells by acidified ethanol. The test was performed in triplicate and the absorbance was measured at $534 \mathrm{~nm}$. Xylitol significantly reduced prodigiosin production. The data shown are the means \pm standard errors. One-way ANOVA test followed by Dunnett's Multiple Comparison test was used for statistical analysis. ${ }^{* * *}: p \leq 0.001$.

\subsection{Xylitol Interferes with Protease Activity}

Skim milk agar was used to assay the effect of xylitol on protease activity. It was found that xylitol completely abolished protease activity (Figure 3).

A)

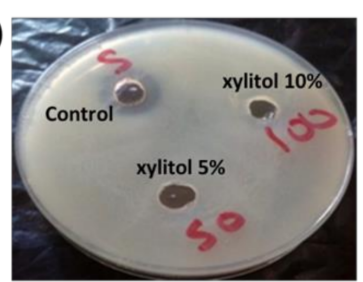

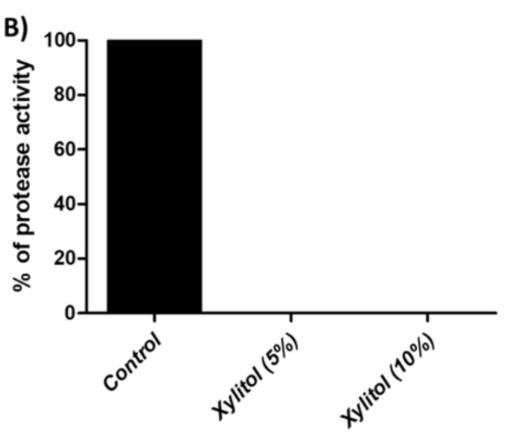

Figure 3. (A) Inhibition of protease activity by the skim milk agar method. The wells made in skim milk LB agar plates were inoculated with aliquots of $(100 \mu \mathrm{L})$ of the supernatants of xylitoltreated and untreated cultures. The clear zones around the wells were measured. The test was performed in triplicate. Xylitol completely blocked the protease activity. (B) The data shown are the means \pm standard errors. One-way ANOVA test followed by Dunnett's Multiple Comparison test was used for statistical analysis.

\subsection{Xylitol Inhibits Swimming and Swarming Motilities}

Prior to host tissues adherence and formation of biofilms, S. marcescens employs swarming and swimming motilities [41]. Xylitol reduced swimming motility by $92.56 \%$ at $5 \%$ and $94.88 \%$ at $10 \%$, and swarming motility by $88.88 \%$ at $5 \%$ and 93.17 at $10 \%$ (Figure 4 ). 

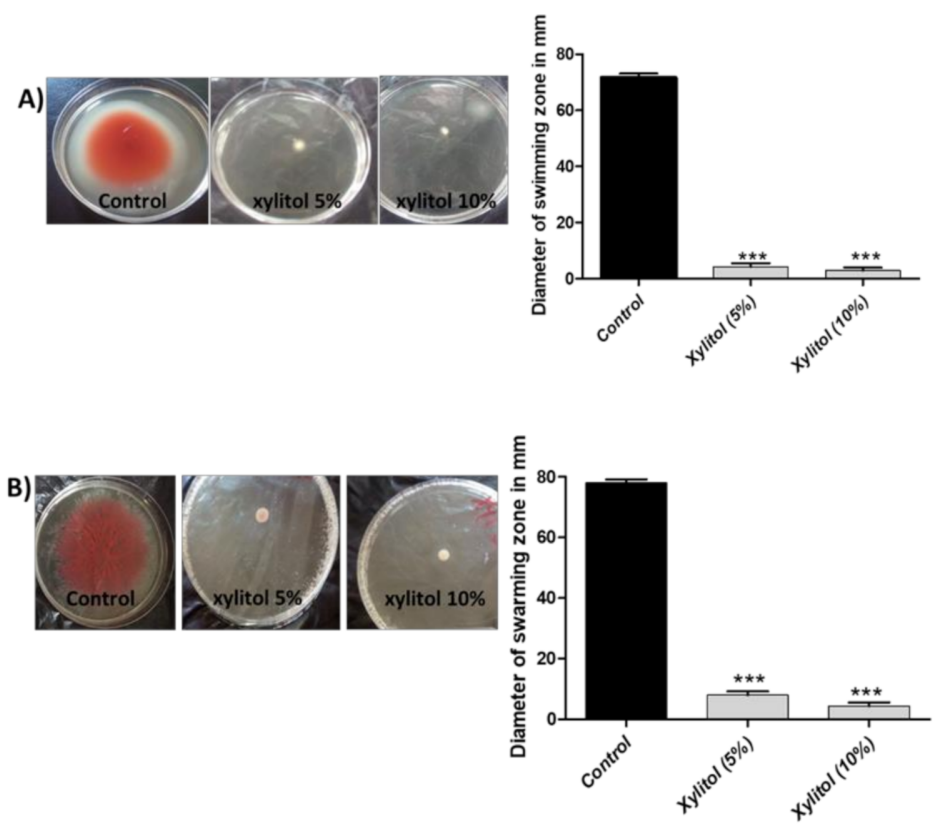

Figure 4. Inhibition of bacterial motility by xylitol. (A) Swimming LB agar plates with xylitol and control plates were center stabbed with $5 \mu \mathrm{L}$ from an overnight culture of $S$. marcescens and the diameter of swimming zones were measured. The test was performed in triplicate. Xylitol significantly reduced swimming motility. The data shown are the means \pm standard errors. Oneway ANOVA test followed by Dunnett's Multiple Comparison test was used for statistical analysis. (B) Swarming LB agar plates with xylitol and control plates were surface inoculated with $5 \mu \mathrm{L}$ from an overnight culture of $S$. marcescens and the diameter of swarming zones were measured. The test was performed in triplicate. Xylitol significantly reduced swarming motility. The data shown are the means \pm standard errors. One-way ANOVA test followed by Dunnett's Multiple Comparison test was used for statistical analysis. ${ }^{* * *}: p \leq 0.001$.

\subsection{Xylitol Increases the Sensitivity to Oxidative Stress}

The hydrogen peroxide disk diffusion assay was used to assess the ability of xylitol to hinder the $S$. marcescens resistance to oxidative stress. In the presence of xylitol, the susceptibility to oxidative stress was enhanced by $16 \%$ for xylitol (5\%) and $23.72 \%$ for xylitol (10\%) (Figure 5).

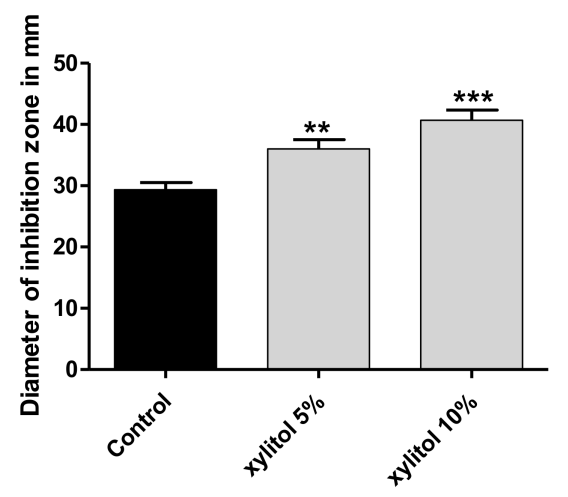

Figure 5. Augmentation of sensitivity to oxidative stress by xylitol. Aliquots of $100 \mu \mathrm{L}$ of overnight culture of $S$. marcescens were spread on the surface of TSA plates with and without xylitol and $10 \mu \mathrm{L}$ of $3 \%$ hydrogen peroxide were added to a filter-paper disk on the plates. The plates were incubated and the inhibition zones were measured. The test was performed in triplicate. Xylitol significantly increased the inhibition zones and the sensitivity to oxidative stress. The data shown are the means \pm standard errors. One-way ANOVA test followed by Dunnett's Multiple Comparison test was used for statistical analysis. ${ }^{* *}: p \leq 0.01$ and ${ }^{* * *}: p \leq 0.001$. 


\subsection{Xylitol Decreased the Expression of S. marcescens Virulence Genes}

The inhibitory activities of xylitol against QS and virulence of $S$. marcescens were confirmed genetically by qRT-PCR. When the expression of virulence genes was evaluated in xylitol-treated and untreated S. marcescens, it was found that the expression levels of all of pigP, shlA, fim A, fim C, bsmB, $r s s B$, flhC, flhD, and $r s m A$ genes significantly decreased in xylitol-treated culture. The down-regulation of the expression of tested genes was fluctuated between 2- to 4-fold reduction in expression levels as indicated in (Figure 6).

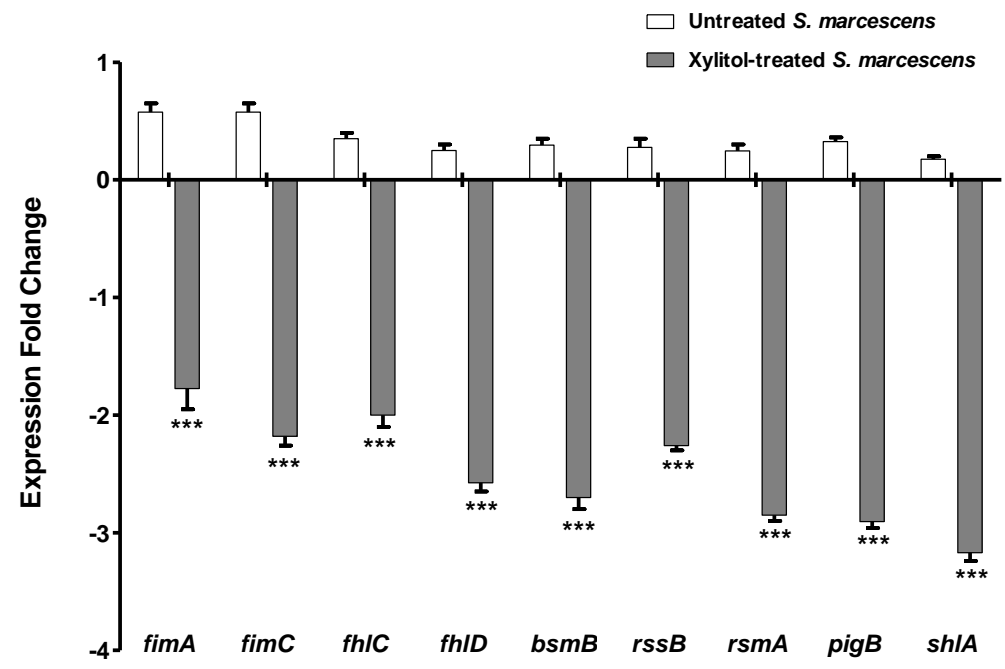

Figure 6. Xylitol decreased the expression of virulence genes of $S$. marcescens. RNA of xylitol-treated and untreated $S$. marcescens cultures was isolated to synthesize cDNA. cDNA was amplified and the change in the expression of each gene were normalized to the rplU gene as the housekeeping gene. The test was performed in triplicate. Xylitol (5\%) significantly reduced the expression levels of fimA, fim $C, f l h C, f l h D, b s m B, r s s B, r s m A, p i g P$, and shlA genes. The data shown are the means \pm standard errors. One-way ANOVA test followed by Dunnett's Multiple Comparison test was used for statistical analysis. ${ }^{* * *}: p \leq 0.001$.

\subsection{In-Vivo Protection Activity of Xylitol Against S. marcescens}

Furtherly, the protective activity of xylitol was in-vivo assessed against $S$. marcescens. Four mouse groups, each comprised of five mice, were assigned and the dead mice were recorded in each group. However, all mice survived in the two negative groups, and only three survived out of five mice in the positive control group. On the other hand, all mice injected with xylitol-treated S. marcescens survived (100\%); this confers a $40 \%$ protection in comparison to the group injected with untreated S. marcescens. The survival of mice was recorded over 5 successive days and plotted by the Kaplan-Meier method where significance $(p<0.05)$ was evaluated using the Log-rank test, GraphPad Prism 8 (Figure 7). Clearly, xylitol significantly decreased the $S$. marcescens capacity to kill mice, ascertained by applying the Log rank test for trend $(p=0.02)$. 


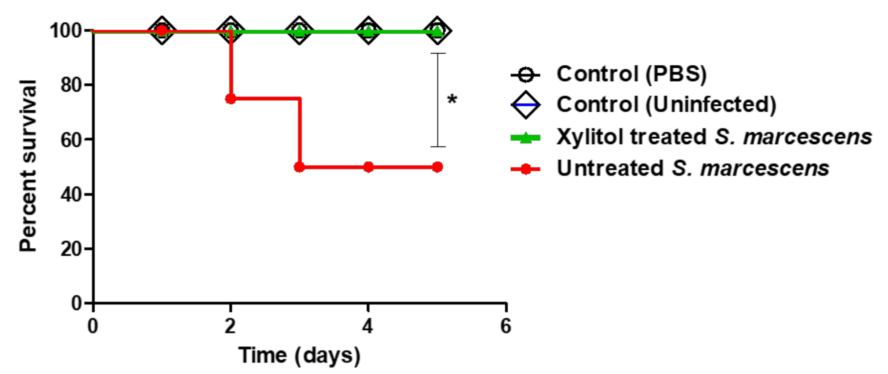

Figure 7. Xylitol protected mice against death by $S$. marcescens. Four mouse groups of healthy mice comprising five mice each were used. In group 1 , mice were intraperitoneally injected with xylitoltreated S. marcescens in sterile PBS, group 2 was injected with untreated bacteria, group 3 was injected with sterile PBS and group 4 was left un-inoculated. Mouse survival in each group was recorded every day over 5 days, plotted using the Kaplan-Meier method, and significance $(p<0.05)$ was calculated using the Log-rank test, GraphPad Prism 5. All mice in groups 3 and 4 (negative control groups) survived, while only $60 \%$ of mice survived (three out of five mice) in the group of the untreated bacteria. In contrast to untreated $S$. marcescens, all mice injected with xylitol-treated $S$. marcescens survived, showing 100\% survival, conferring 40\% protection (Log rank test for trend ${ }^{*} p=0.02$ ).

\subsection{Xylitol Could Bind to Smar Receptor In-Silico}

The binding mode of xylitol was revealed from the performed molecular docking study with SmaR protein. The binding interactions of xylitol and C4-HSL with the target receptor are shown in Figure 8. These interactions included both hydrophobic interaction and hydrogen bonding. The autodock scores for each ligand in addition to the interacting residues are presented (Table 1). Xylitol showed a good ability to bind to SmaR receptors. This might indicate its ability to hinder the binding of the natural ligand to its receptor, and this may result in the inhibition of QS and its regulated virulence factors.
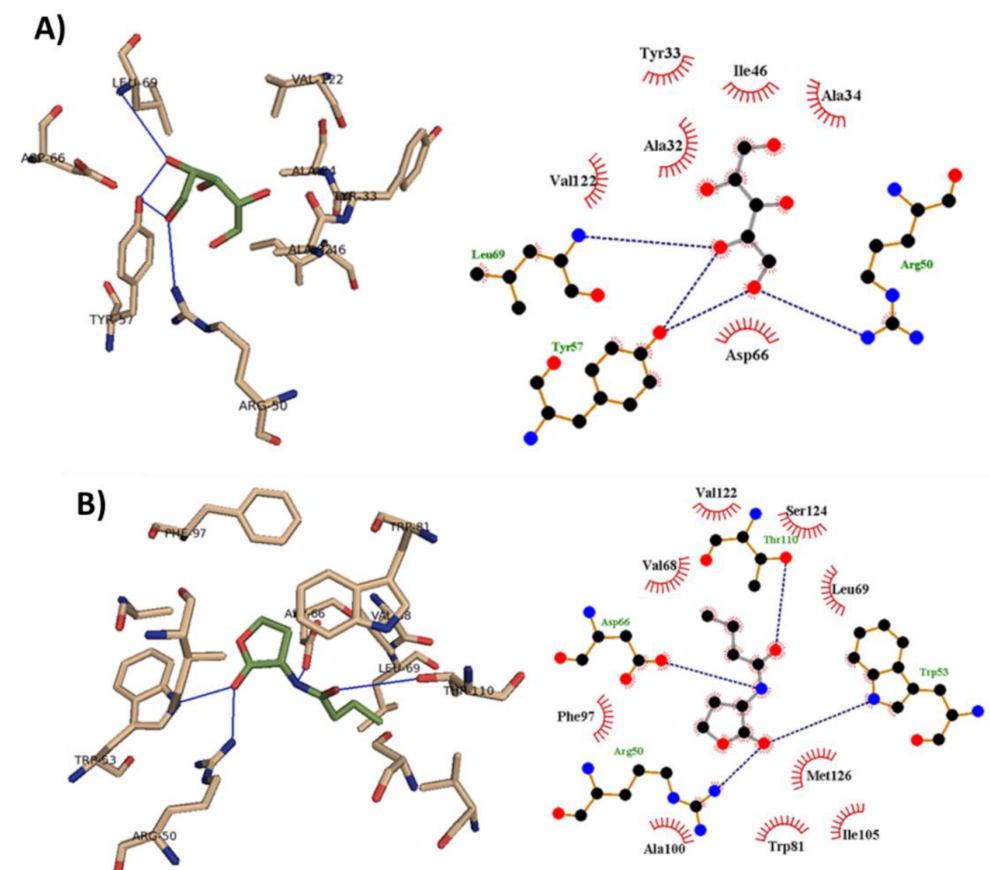

Figure 8. The Molecular docking of (A) Xylitol and (B) C4-HSL into the active site of SmaR protein 3D representation (left) and 2D Schematic interaction (right). Xylitol could bind with SmaR receptors by hydrogen bonding and hydrophobic interaction. 
Table 1. The binding mode of xylitol and natural ligand with the different residues inside the active site of SmaR protein.

\begin{tabular}{cccc}
\hline Ligand & H-Bonding & Hydrophobic Interaction & Autodock Score \\
\hline \multirow{2}{*}{ Xylitol } & Arg 50, Tyr 57, Leu & Ala 32, Tyr 33, Ala 34, Ile 46, Asp 66, & -4.5 \\
& 69 & Val 122 & \\
C4-HSL & Arg 50, Trp 53, Asp & Val 68, Leu 69, Trp 81, Phe 97, Ala 100, & -6.4 \\
& 66, Thr 110 & Ile 105, Val 122, Ser 124, Met 126 & \\
\hline
\end{tabular}

\section{Discussion}

Serratia marcescens is an evolving nosocomial pathogen. Due to its ability to adhere to invasive hospital instrumentation and intravenous tubing, its eradication is difficult by conventional methods [42]. That results in causing various healthcare related infections [43]. Among several gram-negative bacteria, S. marcescens aquires control over their physiological traits using the QS communication machinery. The QS system in S. marcescens' SmaI/SmaR communication system depends on secretion of autoinducers termed homoserine lactones (HSLs). QS orchestrates motility, biofilm formation, production of hydrolytic enzymes, and other virulence factors as lipase, protease, hemolysin, and chitinase [6,44-47].

Bearing in mind the high antibiotic resistance of S. marcescens, it is vital to seek a therapeutic tool other than antibiotics that does not target bacterial growth. Targeting QS can fulfil this purpose because it does not affect cell viability. Instead, QS inhibition can disarm the virulence factors of $S$. marcescens, leaving it eradicable by the immune system [48]. This study was conducted to explore xylitol's ability to interfere with growth and to curtail the virulence regulated by QS in the emerging nosocomial bacterium $S$. marcescens. Xylitol inhibited S. marcescens' growth at $400 \mathrm{mg} / \mathrm{mL}(40 \%)$.

To test the ability of xylitol to interfere with virulence, sub-inhibitory concentrations of xylitol (10\% that represents $1 / 4 \mathrm{MIC}$ and $5 \%$ that represents $1 / 8 \mathrm{MIC})$ were used for the assays. Xylitol significantly reduced biofilm formation by $74.22 \%$ and $81.03 \%$ at $5 \%$ and $10 \%$ of xylitol, respectively. This may be attributed to the anti-adhesive capability of xylitol [49-51]. Swarming and swimming were previously linked to microbial cytotoxicity and required for bacterial adherence as the first step of biofilm formation [52,53]. Serratia marcescens is motile and can move via swimming and swarming types of motility [54]. Xylitol blocked swimming motility by $92.56 \%$ (5\% xylitol) and $94.88 \%$ (10\% xylitol). More or less similar inhibition of swarming motility was achieved ( $88.88 \%$ at $5 \%$ and $93.17 \%$ at $10 \%$ ).

The production of the red characteristic pigment prodigiosin was markedly inhibited by xylitol $(63.42 \%$ at $5 \%$ and $78.47 \%$ at $10 \%)$. This is preliminary evidence of the inhibitory activity of xylitol against QS because it is well reported that prodigiosin is produced under the control of QS [30]. Moreover, xylitol's ability to interfere with protease activity was investigated by the skim milk agar method, and it could completely inhibit protease activity of $S$. marcescens at both $5 \%$ and $10 \%$. Protease activity was reported to have a vital role in human infection and to stimulate inflammatory response [48,55]. Furthermore, resistance to oxidative stress was screened, and xylitol presence enhanced the sensitivity to oxidative stress induced by hydrogen peroxide by $16 \%$ (xylitol 5\%) and 23.72\% (xylitol 10\%).

To confirm the activity of xylitol against $S$. marcescens virulence, the expression rate of some genes involved in these traits under the effect of xylitol (5\%) was measured by quantitative Realtime PCR. As compared to the control untreated culture, the xylitoltreated culture showed significant down-regulation of all tested genes. S. marcescens swarming motility is regulated by flhDC flagellar regulatory operon that encodes the flagellar transcriptional activator FlhD and the flagellar transcriptional regulator FlhC [56,57]. Furthermore, RsmA is considered as a vital component of the swarming regulatory network [41,58-60]. Noteworthy, the flhDC regulator is under the control by the RssAB two-component system $[59,61]$. In the light of the down-regulation of $f l h C, f l h D$, rssB and rsmA by xylitol, it can be understood why it blocked swimming and swarming motilities.

Prodigiosin biosynthesis is encoded by the prodigiosin biosynthetic operon pigA$\mathrm{N}[62,63]$. Xylitol reduced the pigP gene expression, which accounts for the inhibition of 
prodigiosin production. Our study showed that xylitol could downregulate the expression of the shlA gene. S. marcescens secretes the pore-forming toxin ShlA on host cell-to-cell junctions to help invade the host tissues [64].

To establish infections, bacteria adhere to the tissues employing fimbria or pili whose production is also related to biofilm formation in S. marcescens [65]. Type I pili is encoded by the fimABCD operon [66]. Moreover, BsmA and BsmB proteins enhance the production of type I pili in S. marcescens [1,66]. The genes fim $A$ gene encodes fimbrial A subunit protein, while type 1 fimbriae regulatory protein FimB and BsmB protein are encoded by fimB and bsmB genes, respectively. In this study, xylitol downregulated the expression of fimA, fimC and $\operatorname{ssmB}$. This may represent an explanation for the remarkable biofilm-inhibitory activity of xylitol.

To confirm our results, xylitol's ability to protect mice against the virulence of $S$. marcescens was performed by the survival test. Xylitol increased the survival of the mice from $60 \%$ in the control treated group to $100 \%$. The ability of xylitol to bind to SmaR receptors was analyzed by the docking analysis, and it was found that xylitol could interfere with the binding of the natural ligand C4-HSL to SmaR receptors, resulting in possible QS inhibition, and hence anti-virulence activity.

The use of hypertonic sugars to inhibit growth and virulence was previously reported. In a study on multidrug-resistant Pseudomonas aeruginosa clinical isolates, hypertonic glucose inhibited the growth at $30 \%$. Moreover, P. aeruginosa motility, biofilm formation, pyocyanin and elastase were significantly blocked by hypertonic glucose ( $5 \%$ and $10 \%)$. The expression of $P$. aeruginosa QS genes was down-regulated in glucose hypertonic concentration $(20 \%)$. Moreover, the administration of hypertonic glucose $(20 \%)$ increased the survival rates of Galleria mellonella larvae from P. aeruginosa [67]. This may suggest a role for the osmotic stress of xylitol in attenuation of the virulence of $S$. marcescens.

\section{Conclusions}

QS regulates the bacterial virulence and its inhibition can attenuate the virulence of bacteria leading to enhancement of the ability of the immune system to remove the bacteria. The advantage of bacterial virulence inhibition that it is less likely to induce resistance. Xylitol may be a good weapon, or at least an adjunctive weapon, to treat infections caused by $S$. marcescens as it showed growth inhibiting activity and virulence attenuating activity, and it may be further considered in management of wound infection by S. marcescens. In the current study, we are introducing the application of xylitol and other sugar hypertonic preparations as adjunctive therapy for further investigation on treatment of resistant infections.

Author Contributions: Conceptualization, H.A.A. and W.A.H.H.; methodology, H.A.A. and A.N.K.; software, M.A.S. and M.T.K.; validation, R.M., W.E.S. and E.-S.K.; formal analysis, T.S.I., A.N.K. and A.J.A.; investigation, H.A.A. and M.A.S.; resources, A.N.K. and A.J.A.; data curation, T.S.I., M.A.S., A.N.K. and M.T.K.; writing-original draft preparation, H.A.A. and W.A.H.H.; writingreview and editing, W.A.H.H.; visualization, W.A.H.H.; supervision, H.A.A.; project administration, W.A.H.H.; funding acquisition, A.N.K., E.-S.K., R.M. and W.E.S. All authors have read and agreed to the published version of the manuscript.

Funding: The Deanship of Scientific Research (DSR) at King Abdulaziz University, Jeddah, Saudi Arabia has funded this project, under grand no. (RG-16-166-42).

Institutional Review Board Statement: The ethical committee at the Faculty of Pharmacy, Zagazig University permitted in-vivo mice experiments in this work. The experiments were performed in compliance with the guidelines of ARRIVE, in agreement with the U.K. Animals (Scientific Procedures) Act, 1986 and related guidelines (ECAHZU, 6 September 2017).

Informed Consent Statement: This study does not include any studies with human participants.

Conflicts of Interest: The authors declare no conflict of interest. 


\section{References}

1. Ramanathan, S.; Ravindran, D.; Arunachalam, K.; Arumugam, V.R. Inhibition of quorum sensing-dependent biofilm and virulence genes expression in environmental pathogen Serratia marcescens by petroselinic acid. Antonie Van Leeuwenhoek 2018, 111, 501-515. [CrossRef] [PubMed]

2. Us, E.; Kutlu, H.H.; Tekeli, A.; Ocal, D.; Cirpan, S.; Memikoglu, K.O. Wound and soft tissue infections of Serratia marcescens in patients receiving wound care: A health care-associated outbreak. Am. J. Infect. Control. 2017, 45, 443-447. [CrossRef] [PubMed]

3. Posluszny, J.A., Jr.; Conrad, P.; Halerz, M.; Shankar, R.; Gamelli, R.L. Surgical burn wound infections and their clinical implications. J. Burn Care Res. 2011, 32, 324-333. [CrossRef] [PubMed]

4. Church, D.; Elsayed, S.; Reid, O.; Winston, B.; Lindsay, R. Burn wound infections. Clin. Microbiol. Rev. 2006, 19, 403-434. [CrossRef]

5. Fekrirad, Z.; Kashef, N.; Arefian, E. Photodynamic inactivation diminishes quorum sensing-mediated virulence factor production and biofilm formation of Serratia marcescens. World J. Microbiol. Biotechnol. 2019, 35, 191. [CrossRef]

6. Van Houdt, R.; Givskov, M.; Michiels, C.W. Quorum sensing in Serratia. FEMS Microbiol. Rev. 2007, 31, 407-424. [CrossRef]

7. Abbas, H.A.; Hegazy, W.A.H. Repurposing anti-diabetic drug "Sitagliptin" as a novel virulence attenuating agent in Serratia marcescens. PLoS ONE 2020, 15, e0231625. [CrossRef]

8. Williamson, N.R.; Simonsen, H.T.; Ahmed, R.A.; Goldet, G.; Slater, H.; Woodley, L.; Leeper, F.J.; Salmond, G.P. Biosynthesis of the red antibiotic, prodigiosin, in Serratia: Identification of a novel 2-methyl-3-n-amyl-pyrrole (MAP) assembly pathway, definition of the terminal condensing enzyme, and implications for undecylprodigiosin biosynthesis in Streptomyces. Mol. Microbiol. 2005, 56, 971-989. [CrossRef]

9. Stock, I.; Burak, S.; Sherwood, K.J.; Gruger, T.; Wiedemann, B. Natural antimicrobial susceptibilities of strains of 'unusual' Serratia species: S. ficaria, S. fonticola, S. odorifera, S. plymuthica and S. rubidaea. J. Antimicrob. Chemother. 2003, 51, 865-885. [CrossRef]

10. Abbas, H.A.; Hegazy, W.A.H. Targeting the virulence factors of Serratia marcescens by ambroxol. Roum. Arch. Microbiol. Immunol. 2017, 76, 27-32.

11. Abbas, H.A.; Soliman, W.E.; Shaldam, M.A. Perturbation of Quorum Sensing in Pseudomonas aeruginosa by Febuxostat. Adv. Microbiol. 2018, 650-664. [CrossRef]

12. Labbate, M.; Zhu, H.; Thung, L.; Bandara, R.; Larsen, M.R.; Willcox, M.D.; Givskov, M.; Rice, S.A.; Kjelleberg, S. Quorum-sensing regulation of adhesion in Serratia marcescens MG1 is surface dependent. J. Bacteriol. 2007, 189, 2702-2711. [CrossRef]

13. Devi, K.R.; Srinivasan, S.; Ravi, A.V. Inhibition of quorum sensing-mediated virulence in Serratia marcescens by Bacillus subtilis R-18. Microb. Pathog. 2018, 120, 166-175. [CrossRef]

14. Rasko, D.A.; Sperandio, V. Anti-virulence strategies to combat bacteria-mediated disease. Nat. Rev. Drug Discov. 2010, 9, 117-128. [CrossRef]

15. Hegazy, W.A.H.; Khayat, M.T.; Ibrahim, T.S.; Nassar, M.S.; Bakhrebah, M.A.; Abdulaal, W.H.; Alhakamy, N.A.; Bendary, M.M. Repurposing Anti-diabetic Drugs to Cripple Quorum Sensing in Pseudomonas aeruginosa. Microorganisms 2020, 8, 1285. [CrossRef]

16. Chirife, J.; Scarmato, G.; Herszage, L. Scientific basis for use of granulated sugar in treatment of infected wounds. Lancet 1982, 1, 560-561. [CrossRef]

17. Zhou, D.P.; Lu, L.Q.; Mao, X.L. Insulin and hyperosmotic glucose solution external used for treating pressure sore. Hunan Yi Ke Da Хие Хие Вао 2001, 26, 475-476.

18. Rabago, D.; Zgierska, A.; Fortney, L.; Kijowski, R.; Mundt, M.; Ryan, M.; Grettie, J.; Patterson, J.J. Hypertonic dextrose injections (prolotherapy) for knee osteoarthritis: Results of a single-arm uncontrolled study with 1-year follow-up. J. Altern. Complement. Med. 2012, 18, 408-414. [CrossRef]

19. Tapiainen, T.; Kontiokari, T.; Sammalkivi, L.; Ikaheimo, I.; Koskela, M.; Uhari, M. Effect of xylitol on growth of Streptococcus pneumoniae in the presence of fructose and sorbitol. Antimicrob. Agents Chemother. 2001, 45, 166-169. [CrossRef]

20. Trahan, L.; Mouton, C. Selection for Streptococcus mutans with an altered xylitol transport capacity in chronic xylitol consumers. J. Dent. Res. 1987, 66, 982-988. [CrossRef]

21. Dowd, S.E.; Sun, Y.; Smith, E.; Kennedy, J.P.; Jones, C.E.; Wolcott, R. Effects of biofilm treatments on the multi-species Lubbock chronic wound biofilm model. J. Wound Care 2009, 18, 508-512. [CrossRef] [PubMed]

22. Ammons, M.C.; Ward, L.S.; James, G.A. Anti-biofilm efficacy of a lactoferrin/xylitol wound hydrogel used in combination with silver wound dressings. Int. Wound J. 2011, 8, 268-273. [CrossRef] [PubMed]

23. Mukherji, R.; Joshi-Navare, K.; Prabhune, A. Crystalline xylitol production by a novel yeast, Pichia caribbica (HQ222812), and its application for quorum sensing inhibition in gram-negative marker strain Chromobacterium violaceum CV026. Appl. Biochem. Biotechnol. 2013, 169, 1753-1763. [CrossRef] [PubMed]

24. Abbas, H.A.; Serry, F.M.; El-Masry, E.M. Combating Pseudomonas aeruginosa biofilms by potential biofilm inhibitors. Asian J. Res. Pharm. Sci. 2012, 2, 66-72.

25. Hegazy, W.A.H.; Khayat, M.T.; Ibrahim, T.S.; Youns, M.; Mosbah, R.; Soliman, W.E. Repurposing of antidiabetics as Serratia marcescens virulence inhibitors. Braz J. Microbiol. 2021, 52, 627-638. [CrossRef]

26. Hegazy, W.A.H. Diclofenac inhibits virulence of Proteus mirabilis isolated from diabetic foot ulcer. Afr. J. Microbiol. Res. 2016, 10, 733-743. [CrossRef] 
27. Issac Abraham, S.V.; Palani, A.; Ramaswamy, B.R.; Shunmugiah, K.P.; Arumugam, V.R. Antiquorum sensing and antibiofilm potential of Capparis spinosa. Arch. Med. Res. 2011, 42, 658-668. [CrossRef]

28. Abd El-Hamid, M.I.; El-Naenaeey, E.-s.Y.; Kandeel, T.M.; Hegazy, W.A.H.; Mosbah, R.A.; Nassar, M.S.; Bakhrebah, M.A.; Abdulaal, W.H.; Alhakamy, N.A.; Bendary, M.M. Promising Antibiofilm Agents: Recent Breakthrough against Biofilm Producing Methicillin-Resistant Staphylococcus aureus. Antibiotics 2020, 9, 667. [CrossRef]

29. Sarkar, R.; Chaudhary, S.K.; Sharma, A.; Yadav, K.K.; Nema, N.K.; Sekhoacha, M.; Karmakar, S.; Braga, F.C.; Matsabisa, M.G.; Mukherjee, P.K.; et al. Anti-biofilm activity of Marula-A study with the standardized bark extract. J. Ethnopharmacol. 2014, 154, 170-175. [CrossRef]

30. Morohoshi, T.; Shiono, T.; Takidouchi, K.; Kato, M.; Kato, N.; Kato, J.; Ikeda, T. Inhibition of quorum sensing in Serratia marcescens AS-1 by synthetic analogs of N-acylhomoserine lactone. Appl. Environ. Microbiol. 2007, 73, 6339-6344. [CrossRef]

31. Hassett, D.J.; Schweizer, H.P.; Ohman, D.E. Pseudomonas aeruginosa sodA and sodB mutants defective in manganese- and iron-cofactored superoxide dismutase activity demonstrate the importance of the iron-cofactored form in aerobic metabolism. J. Bacteriol. 1995, 177, 6330-6337. [CrossRef]

32. Salini, R.; Pandian, S.K. Interference of quorum sensing in urinary pathogen Serratia marcescens by Anethum graveolens. Pathog. Dis. 2015, 73, ftv038. [CrossRef]

33. Askoura, M.; Hegazy, W.A.H. Ciprofloxacin interferes with Salmonella Typhimurium intracellular survival and host virulence through repression of Salmonella pathogenicity island-2 (SPI-2) genes expression. Pathog. Dis. 2020, 78, ftaa011. [CrossRef]

34. Bendary, M.M.; Ibrahim, D.; Mosbah, R.A.; Mosallam, F.; Hegazy, W.A.H.; Awad, N.F.S.; Alshareef, W.A.; Alomar, S.Y.; Zaitone, S.A.; Abd El-Hamid, M.I. Thymol Nanoemulsion: A New Therapeutic Option for Extensively Drug Resistant Foodborne Pathogens. Antibiotics 2020, 10, 25. [CrossRef]

35. Kim, H.S.; Lee, S.H.; Byun, Y.; Park, H.D. 6-Gingerol reduces Pseudomonas aeruginosa biofilm formation and virulence via quorum sensing inhibition. Sci. Rep. 2015, 5, 8656. [CrossRef]

36. Waterhouse, A.; Bertoni, M.; Bienert, S.; Studer, G.; Tauriello, G.; Gumienny, R.; Heer, F.T.; de Beer, T.A.P.; Rempfer, C.; Bordoli, L.; et al. SWISS-MODEL: Homology modelling of protein structures and complexes. Nucleic Acids Res. 2018, 46, W296-W303. [CrossRef]

37. Zhang, R.G.; Pappas, K.M.; Brace, J.L.; Miller, P.C.; Oulmassov, T.; Molyneaux, J.M.; Anderson, J.C.; Bashkin, J.K.; Winans, S.C.; Joachimiak, A. Structure of a bacterial quorum-sensing transcription factor complexed with pheromone and DNA. Nature 2002, 417, 971-974. [CrossRef]

38. Trott, O.; Olson, A.J. AutoDock Vina: Improving the speed and accuracy of docking with a new scoring function, efficient optimization, and multithreading. J. Comput. Chem. 2010, 31, 455-461. [CrossRef]

39. Sanner, M.F. Python: A programming language for software integration and development. J. Mol. Graph. Model. 1999, 17, 57-61.

40. Laskowski, R.A.; Swindells, M.B. LigPlot+: Multiple ligand-protein interaction diagrams for drug discovery. J. Chem. Inf. Model. 2011, 51, 2778-2786. [CrossRef]

41. Lin, C.S.; Tsai, Y.H.; Chang, C.J.; Tseng, S.F.; Wu, T.R.; Lu, C.C.; Wu, T.S.; Lu, J.J.; Horng, J.T.; Martel, J.; et al. An iron detection system determines bacterial swarming initiation and biofilm formation. Sci. Rep. 2016, 6, 36747. [CrossRef]

42. Rodrigues, A.P.; Holanda, A.R.; Lustosa, G.P.; Nobrega, S.M.; Santana, W.J.; Souza, L.B.; Coutinho, H.D. Virulence factors and resistance mechanisms of Serratia marcescens. A short review. Acta Microbiol. Immunol. Hung. 2006, 53, 89-93. [CrossRef]

43. Jones, R.N. Microbial etiologies of hospital-acquired bacterial pneumonia and ventilator-associated bacterial pneumonia. Clin. Infect. Dis. 2010, 51, S81-S87. [CrossRef]

44. Mangwani, N.; Kumari, S.; Das, S. Bacterial biofilms and quorum sensing: Fidelity in bioremediation technology. Biotechnol. Genet. Eng. Rev. 2016, 32, 43-73. [CrossRef]

45. Zhou, J.; Lyu, Y.; Richlen, M.; Anderson, D.M.; Cai, Z. Quorum sensing is a language of chemical signals and plays an ecological role in algal-bacterial interactions. CRC Crit. Rev. Plant. Sci. 2016, 35, 81-105. [CrossRef]

46. Srinivasan, R.; Mohankumar, R.; Kannappan, A.; Karthick Raja, V.; Archunan, G.; Karutha Pandian, S.; Ruckmani, K.; Veera Ravi, A. Exploring the Anti-quorum Sensing and Antibiofilm Efficacy of Phytol against Serratia marcescens Associated Acute Pyelonephritis Infection in Wistar Rats. Front. Cell Infect. Microbiol. 2017, 7, 498. [CrossRef]

47. Hegazy, W.A.H.; Abbas, H.A. Evaluation of the role of SsaV 'Salmonella pathogenicity island-2 dependent type III secretion system components on the virulence behavior of Salmonella enterica serovar Typhimurium. Afr. J. Biotechnol. 2017, 16, 718-726. [CrossRef]

48. Coulthurst, S.J.; Williamson, N.R.; Harris, A.K.P.; Spring, D.R.; Salmond, G.P.C. Metabolic and regulatory engineering of Serratia marcescens: Mimicking phage-mediated horizontal acquisition of antibiotic biosynthesis and quorum-sensing capacities. Microbiology 2006, 152, 1899-1911. [CrossRef]

49. Pereira, A.D.F.F.; Caldana, M.L.d.S.T.; de Andrade Moreira Machado, M.A.; Buzalaf, M.A.R. Use of xylitol for prevention of acute otitis media. Arq. Int. Otorrinolaringol. 2009, 13, 87-92.

50. Da Silva, A.F.S.S.; Oliveira, M.G.; Raposo, N.R.B. Inibição in vitro da aderência de enteropatógenos pelo xilitol. Rev. Ciênc. Méd. Biol. 2010, 9, 46-53. [CrossRef]

51. Silva, A.F.D.S.E.; Ferreira, A.S.; Oliveira, M.S.; Silva, S.S.D.; Raposo, N.R.B. In vitro inhibition of adhesion of Escherichia coli strains by Xylitol. Braz. Arch. Biol. Technol. 2011, 54, 235-241. [CrossRef] 
52. Kirov, S.M. Bacteria that express lateral flagella enable dissection of the multifunctional roles of flagella in pathogenesis. Fems Microbiol. Lett. 2003, 224, 151-159. [CrossRef]

53. Rice, S.A.; Koh, K.S.; Queck, S.Y.; Labbate, M.; Lam, K.W.; Kjelleberg, S. Biofilm formation and sloughing in Serratia marcescens are controlled by quorum sensing and nutrient cues. J. Bacteriol. 2005, 187, 3477-3485. [CrossRef] [PubMed]

54. Kearns, D.B. A field guide to bacterial swarming motility. Nat. Rev. Microbiol. 2010, 8, 634-644. [CrossRef]

55. Kida, Y.; Inoue, H.; Shimizu, T.; Kuwano, K. Serratia marcescens serralysin induces inflammatory responses through proteaseactivated receptor 2. Infect. Immun. 2007, 75, 164-174. [CrossRef]

56. Nicholson, W.L.; Leonard, M.T.; Fajardo-Cavazos, P.; Panayotova, N.; Farmerie, W.G.; Triplett, E.W.; Schuerger, A.C. Complete Genome Sequence of Serratia liquefaciens Strain ATCC 27592. Genome Announc. 2013, 1, e00548-13. [CrossRef]

57. Iguchi, A.; Nagaya, Y.; Pradel, E.; Ooka, T.; Ogura, Y.; Katsura, K.; Kurokawa, K.; Oshima, K.; Hattori, M.; Parkhill, J.; et al. Genome evolution and plasticity of Serratia marcescens, an important multidrug-resistant nosocomial pathogen. Genome Biol. Evol. 2014, 6, 2096-2110. [CrossRef]

58. Liaw, S.J.; Lai, H.C.; Ho, S.W.; Luh, K.T.; Wang, W.B. Role of RsmA in the regulation of swarming motility and virulence factor expression in Proteus mirabilis. J. Med. Microbiol. 2003, 52, 19-28. [CrossRef]

59. Lin, C.S.; Horng, J.T.; Yang, C.H.; Tsai, Y.H.; Su, L.H.; Wei, C.F.; Chen, C.C.; Hsieh, S.C.; Lu, C.C.; Lai, H.C. RssAB-FlhDC-ShlBA as a major pathogenesis pathway in Serratia marcescens. Infect. Immun. 2010, 78, 4870-4881. [CrossRef]

60. Askoura, M.; Youns, M.; Halim Hegazy, W.A. Investigating the influence of iron on Campylobacter jejuni transcriptome in response to acid stress. Microb. Pathog. 2020, 138, 103777. [CrossRef]

61. Wei, C.F.; Tsai, Y.H.; Tsai, S.H.; Lin, C.S.; Chang, C.J.; Lu, C.C.; Huang, H.C.; Lai, H.C. Cross-talk between bacterial two-component systems drives stepwise regulation of flagellar biosynthesis in swarming development. Biochem. Biophys. Res. Commun. 2017, 489, 70-75. [CrossRef]

62. Stella, N.A.; Fender, J.E.; Lahr, R.M.; Kalivoda, E.J.; Shanks, R.M. The LysR Transcription Factor, HexS, Is Required for Glucose Inhibition of Prodigiosin Production by Serratia marcescens. Adv. Microbiol. 2012, 2, 10.4236/aim.2012.24065. [CrossRef]

63. Brothers, K.M.; Stella, N.A.; Romanowski, E.G.; Kowalski, R.P.; Shanks, R.M. EepR Mediates Secreted-Protein Production, Desiccation Survival, and Proliferation in a Corneal Infection Model. Infect. Immun. 2015, 83, 4373-4382. [CrossRef]

64. Reboud, E.; Bouillot, S.; Patot, S.; Beganton, B.; Attree, I.; Huber, P. Pseudomonas aeruginosa ExlA and Serratia marcescens ShlA trigger cadherin cleavage by promoting calcium influx and ADAM10 activation. PLoS Pathog. 2017, 13, e1006579. [CrossRef]

65. Shanks, R.M.; Stella, N.A.; Brothers, K.M.; Polaski, D.M. Exploitation of a "hockey-puck" phenotype to identify pilus and biofilm regulators in Serratia marcescens through genetic analysis. Can. J. Microbiol. 2016, 62, 83-93. [CrossRef]

66. Shanks, R.M.; Stella, N.A.; Kalivoda, E.J.; Doe, M.R.; O'Dee, D.M.; Lathrop, K.L.; Guo, F.L.; Nau, G.J. A Serratia marcescens OxyR homolog mediates surface attachment and biofilm formation. J. Bacteriol. 2007, 189, 7262-7272. [CrossRef]

67. Chen, T.; Xu, Y.; Xu, W.; Liao, W.; Xu, C.; Zhang, X.; Cao, J.; Zhou, T. Hypertonic glucose inhibits growth and attenuates virulence factors of multidrug-resistant Pseudomonas aeruginosa. BMC Microbiol. 2020, 20, 203. [CrossRef] 